African J. Biol. Sci., 17 (1): 111-116 (2021)

ISSN 1687-4870

www.ajbs.journals.ekb.eg

\author{
e- ISSN 2314-5501 (online) \\ E.mail: aasdjournal@yahoo.com
}

\title{
An annotated checklist of family Hydraenidae (Coleoptera: Staphylinoidea) in the North Africa
}

Ahmed R. Ismaieel ${ }^{1}$, Hassan H. Fadl ${ }^{2}$ and Gawhara M. M. Abu El-hassan ${ }^{3}$

Department of Entomology, Faculty of Science, Ain Shams University, Abbassia- Cairo, Egypt.

${ }^{1}$ ahmedreda@ sci.asu.edu.eg; ${ }^{2}$ fadlaly@yahoo.com; ${ }^{3}$ gawhara_magdy@ sci.asu.edu.eg

Received: May 10, 2021; Accepted: May 21, 2021.; Available online: June 3, 2021.

\begin{abstract}
Data from previous literature were used to compile a checklist of the of north African hydraenid beetles. Members of Hydraenidae are mostly $0.5-3 \mathrm{~mm}$ in length and genitalia of different species under the same taxonomic group are almost indistinguishable. The external morphology of several taxa, e.g., Ochthebiinae, is largely variable. Mostly all the described genera or subgenera are with uncertain relationships in addition to the presence of many assigned but undescribed species. Here we summarize the recorded species of family Hydraenidae in the north Africa. The species distribution in the Palearctic region is provided. According to the analysis of literature a total of 15, 15, 61, 39 species were recorded in Algeria, Egypt, Morocco, Tunisia respectively. The genus Ochthebius was represented by 15, 13, 36, 16 species, while Hydraena by 19, 1, 15, 12 species, and Limnebius species was represented by 3,1, 12, 6 species in Algeria Egypt, Morocco, Tunisia respectively.
\end{abstract}

Keywords: Minute Moss Beetles, checklist, distribution, Palearctic, Egypt, Morocco, Tunisia, Algeria

\section{INTRODUCTION}

Hydraenidae (minute moss beetles) is an old and well-known aquatic beetles' family (Ponomarenko \& Prokin, 2015), which belongs to suborder Polyphaga, Infraorder Staphyliniformia, Superfamily Staphylinoidea, order Coleoptera (Hansen, 1987). Approximately 1600 species under 42 genera have been identified within this family (Ślipiński et al., 2011). The Palearctic region itself about 800 species (Jäch, 2004). About 1000 hydraenid species in 22 genera are undescribed yet (Epler, 2010; Jäch \& Balke, 2008). In this work, a checklist of the Hydraenidae from Algeria, Egypt, Morocco, and Tunisia is provided with the valid names.

MATERIALS AND METHODS Biogeography (Fig. 1)

Algeria (AL): is located in the Maghreb region of North Africa, between Tunisia (East), Morocco (West), on 2,381,741 square kilometers area.

Egypt (EG): is located in North Africa, between The Reda Sea (East), Libya (West), one million square kilometers area.

Morocco (MO): is located in the Maghreb region of North Africa, between Algeria (East), The Atlantic Ocean (West), on 710,850 square kilometers area.

Tunisia (TU): is located in the Maghreb region of North Africa, between Libya (East), Algeria (West), on 163,610 square area.

The present study includes all the valid names based on the recent catalogues, and literature of Hansen, 1998; Jäch, 2004; Touaylia et al., 2011 Salah et al., 2014; Mabrouki et al., 2018; Lamine et al., 2019. 


\section{Ahmed R. Ismaieel et al.}

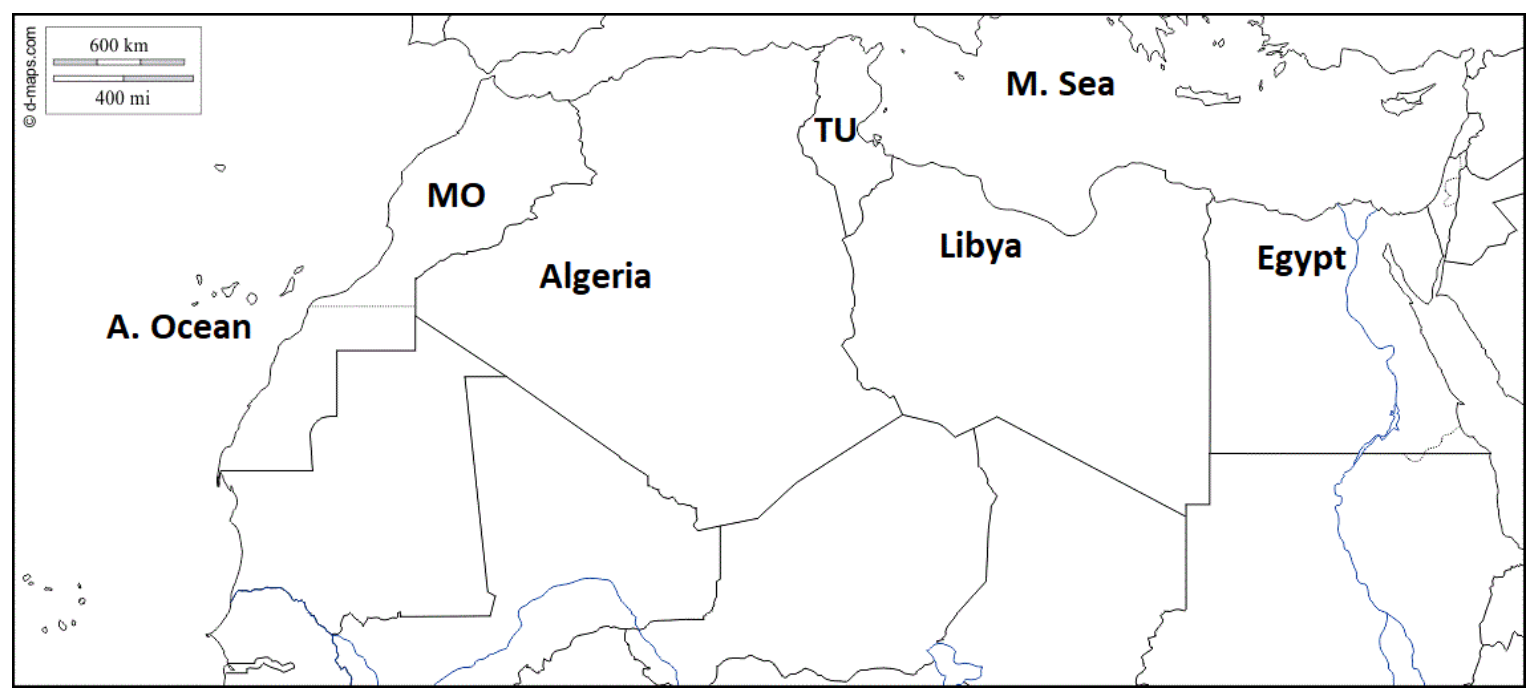

Fig. 1. Map shows the North African Countries (Algeria, MO: Morocco, TU: Tunisia, Libya, Egypt).

\section{RESULTS AND DISCUSSION}

The North African Hydraenidae beetle's family is divided into two Subfamilies; Hydraeninae and Ochthebiinae and it includes three genera; Hydraena; Limnebius and Ochthebius. Despite the huge number of Hydraenidae species all over the world, it is represented in the north Africa only by 97 species, without any previous record of these beetles from Libya (Table 1). So Hydraenidae taxonomic and ecological studies need to be intensive to get the precise image of the number of genera and species of this family, and to protect their aquatic habitats from destruction. There are many taxonomic dilemmas in the taxonomic status of this family such as species complexes, genitalia similarities, and morphometric character variation among individuals of the same species, so beetle taxonomists should spot the light on this family in the future.

Table 1. The North African Hydraenidae beetle spcies.

\begin{tabular}{|l|l|l|l|l|l|}
\hline & Species & AL & EG & MO & TU \\
\hline $\mathbf{1}$ & Hydraena africana Kuwert, 1888 & - & - & + & - \\
\hline $\mathbf{2}$ & H. algerina Kaddouri, 1992 & + & - & - & - \\
\hline $\mathbf{3}$ & H. allomorpha Lagar \& Fresneda, 1990 & - & - & + & - \\
\hline $\mathbf{4}$ & H. antiatlantica Jäch, Aguilera \& Hernando, 1998 & - & - & + & - \\
\hline $\mathbf{5}$ & H. atrata Desbrochers d Loges, 1891 & + & - & + & + \\
\hline $\mathbf{6}$ & H. audisioi Jäch, 1992 & + & - & - & - \\
\hline $\mathbf{7}$ & H. bedeli Berthelemy, 1992 & + & - & - & - \\
\hline $\mathbf{8}$ & H. bisulcata Rey, 1884 & - & - & + & - \\
\hline $\mathbf{9}$ & H. capta d'Orchymont, 1936 & - & - & + & - \\
\hline
\end{tabular}


An annotated checklist of family Hydraenidae (Coleoptera: Staphylinoidea) in the North Africa

\begin{tabular}{|c|c|c|c|c|c|}
\hline 10 & H. chobauti Guillebeau, $1896 \mathrm{~b}$ & + & - & - & - \\
\hline 11 & H. cordata L. W. Schaufuss, 1883 & + & - & + & + \\
\hline 12 & H. exasperate d'Orchymont, 1935 & - & - & + & - \\
\hline 13 & H. explanata Pic, 1905 & + & - & - & - \\
\hline 14 & H. hernandoi Fresneda\&Lagar, 1990 & + & - & + & - \\
\hline 15 & H. kocheri Berthelemy, 1992 & + & - & + & - \\
\hline 16 & H. kroumiriana Kaddouri, 1992 & - & - & - & + \\
\hline 17 & H. leprieuri S.C Deville, 1905 & + & - & - & + \\
\hline 18 & H. mouzaiensis S.C Deville, 1909 & + & - & - & + \\
\hline 19 & H. numidica S.C Deville, 1905 & + & - & - & + \\
\hline 20 & H. pallidula S.C Deville, 1909 & + & - & - & - \\
\hline 21 & H. pici S.C Deville, 1905 & + & - & - & + \\
\hline 22 & H. quadricollis Wollaston 1864 & + & + & + & + \\
\hline 23 & H. rigua d'Orchymont, 1931 & + & - & + & + \\
\hline 24 & H. riberai Jäch, Aguilera \& Hernando, 1998 & - & - & + & - \\
\hline 25 & H. rivularis Guillebeau, 1896 & + & - & - & + \\
\hline 26 & H. scabrosad'Orchymont, 1931 & + & - & + & + \\
\hline 27 & H. testacea Curtis, 1830 & + & - & + & + \\
\hline 28 & Limnebius aguilerai Ribera \& Millan, 1998 & - & - & + & - \\
\hline 29 & L. alibeii Hernando, Aguilera \& Ribera, 1999 & - & - & + & - \\
\hline 30 & L. bacchus Balfour-Browne, 1979 & - & - & + & - \\
\hline 31 & L. evanescens Kiesenwetter, 1866 & + & - & + & - \\
\hline 32 & L. extraneus d'Orchymont, 1938 & - & - & + & - \\
\hline 33 & L. fretalis Peyerimhoff, 1913 & - & - & + & - \\
\hline 34 & L. furcatus Baudi di Selve, 1872 & - & - & + & + \\
\hline 35 & L. irmelae lach, 1993 & - & - & - & + \\
\hline 36 & L. kocheri Balfour-Browne, 1979 & - & - & + & - \\
\hline 37 & L. maurus Balfour-Browne, 1979 & - & - & + & - \\
\hline 38 & L. mesatlanticus Thery, 1933 & - & - & + & - \\
\hline 39 & L. nitifarus d' Orchymont, 1938 & + & - & - & + \\
\hline 40 & L. oblongus Rey, 1883 & - & - & + & - \\
\hline 41 & L. perparvulus Rey, 1884 & - & - & - & + \\
\hline 42 & L. pilicauda Guillebeau, 1896 & + & - & + & + \\
\hline 43 & L. sanctimontis Jäch, 1993 & - & + & - & - \\
\hline 44 & L. theryi Guillebeau, 1891 & - & - & - & + \\
\hline 45 & Ochthebius abeillei Guillebeau, 1896 & - & + & + & - \\
\hline 46 & O. aeneus Stephens, 1835 & - & - & + & + \\
\hline 47 & O. auropallens Fairmaire, 1879 & + & - & + & + \\
\hline 48 & O. anxifer Balfour-Browne, 1979 & - & - & + & - \\
\hline 49 & O. bifoveolatus Waltl, 1835 & + & - & + & - \\
\hline 50 & O. bonnairei Guillebeau, $1896 \mathrm{~b}$ & + & - & + & + \\
\hline
\end{tabular}


Ahmed R. Ismaieel et al.

\begin{tabular}{|c|c|c|c|c|c|}
\hline 51 & O. corrugates Rosenhauer, 1856 & - & + & - & - \\
\hline 52 & O. cuprescens Guillebeau, 1893 & - & - & + & - \\
\hline 53 & O. difficilis Mulsant, 1844 & + & - & + & - \\
\hline 54 & O. dilatatus Stephens, 1829 & + & - & - & + \\
\hline 55 & O. exaratus Mulsant, 1844 & - & + & - & - \\
\hline 56 & O. eyreiJäch, 1990 & - & - & - & + \\
\hline 57 & O. figueroi Garrido Gonzalez, 1990 & - & - & + & - \\
\hline 58 & O. fossulatus Mulsant, 1844 & - & - & + & + \\
\hline 59 & O. gauthieri Peyerimhoff, 1924 & + & - & + & - \\
\hline 60 & O. grandipennis Fairmaire, 1879 & + & - & - & - \\
\hline 61 & O. kieneri Jäch, 1999 & + & - & - & - \\
\hline 62 & O. lanarotis Ferro, 1985 & - & - & + & - \\
\hline 63 & O. libertarius Aguilera, Ribera \& Hernando, 1998 & - & - & + & - \\
\hline 64 & O. lividipennis Peyron, 1858 & - & + & + & - \\
\hline 65 & O. mauretanicus Jäch, 1990 & - & - & + & - \\
\hline 66 & O. marinus Paykull, 1798 & - & - & + & - \\
\hline 67 & O. maroccanus Jäch,1992 & - & - & + & - \\
\hline 68 & O. mediterraneus Ienieștea, 1988 & - & - & + & + \\
\hline 69 & O. meridionalis Rey, 1885 & - & + & + & - \\
\hline 70 & O. merinidicus Ferro, 1985 & - & - & - & + \\
\hline 71 & O. minerviussemechonitis Jäch, 1998 & - & + & - & - \\
\hline 72 & O. nanus Stephens, 1829 & - & - & + & - \\
\hline 73 & O. normandi Jäch, 1992 & - & - & - & + \\
\hline 74 & O. notabilis Rosenhauer, 1856 & - & - & + & - \\
\hline 75 & O. perpusillus Ferro, 1985 & - & - & + & - \\
\hline 76 & O. pilosus Waltl, 1835 & + & + & + & - \\
\hline 77 & O. poweri $\mathrm{Rey}, 1870$ & + & - & - & - \\
\hline 78 & O. praetermissus Jäch, 1991 & + & - & + & + \\
\hline 79 & O. punctatus Stephens, 1829 & - & - & + & + \\
\hline 80 & O. quadrifossulatus Waltl, 1835 & - & - & + & - \\
\hline 81 & O. quadricollis Mulsant, 1844 & + & - & + & - \\
\hline 82 & O. quadrifoveolatus Wollaston, 1854 & + & + & + & - \\
\hline 83 & O. ragusaeKuwert, 1887 & - & + & - & - \\
\hline 84 & O. recurvatus Jäch, 1991 & - & - & - & + \\
\hline 85 & O. salinator Peyerimhoff, 1924 & + & + & + & - \\
\hline 86 & O. semisericeus S.C Deville, 1914 & - & - & + & - \\
\hline 87 & O. serratus Rosenhauer, 1856 & - & - & + & - \\
\hline 88 & O. silfverbergi Jäch. 1992 & - & - & - & + \\
\hline 89 & O. subpictussubpictus Wollaston, 1857 & - & + & + & - \\
\hline 90 & O. subinteger Mulsant and Rey, 1861 & - & - & + & - \\
\hline 91 & O. tacapasensis Ferro, 1983 & - & - & + & - \\
\hline
\end{tabular}


An annotated checklist of family Hydraenidae (Coleoptera: Staphylinoidea) in the North Africa

\begin{tabular}{|l|l|l|l|l|l|}
$\mathbf{9 2}$ & O. thermalis Janssens, 1965 & - & + & - & + \\
\hline $\mathbf{9 3}$ & O. tivelunus Ferro, 1984 & - & - & + & - \\
\hline $\mathbf{9 4}$ & O. tunisicus Jäch, 1997 & - & - & - & + \\
\hline $\mathbf{9 5}$ & O. velutinus Fainnaire, 1883 & + & - & - & - \\
\hline $\mathbf{9 6}$ & O. viridescens Ieniştea, 1988 & - & + & + & + \\
\hline $\mathbf{9 7}$ & O. wewalkai Jäch, 1984 & - & + & - & - \\
\hline
\end{tabular}

\section{REFERENCES}

Epler, J.H. (2010). The Water Beetles of Florida: an identification manual for the families Chrysomelidae, Curculionidae, Dryopidae, Dytiscidae, Elmidae, Gyrinidae, Haliplidae, Helophoridae, Hydraenidae, Hydrochidae, Hydrophilidae, Noteridae, Psephenidae, Ptilodactylidae and Scirtidae. Tallahassee: State of Florida Department of Environmental Protection, Division of Environmental Assessment and Restoration, $414 \mathrm{p}$.

Hansen, M. (1987). The Hydrophiloidea (Coleoptera) of Fennoscandia and Denmark. Fauna Entomologica Scandinavica. 18: 1-254.

Jäch, M. A., 2004. Hydraenidae. In: Löbl, I. \& Smetana, A. (Eds.), Catalogue of Palaearctic Coleoptera. Vol. 2. Apollo Books, Stenstrup, 102-122.

Jäch, M.A. and Balke, M. (2008). Global diversity of water beetles (Coleoptera) in freshwater. Hydrobiologia, 595, 419442.http://dx.doi.org/10.1007/s10750 -007-9117-y

Lamine, S.; Lounaci, A. and Bennas, N. (2019). Biodiversity and chorology of aquatic beetles (Coleoptera: Elmidae and Hydraenidae) in Kabylia (central-north Algeria). New records and updates.Zootaxa, 4700(1): 102-116. Doi: https://doi.10.11646/zootaxa.4700.1. $\underline{5}$

Ponomarenko, A. and Prokin, A. (2015). Review of paleontological data on the evolution of aquatic beetles (Coleoptera). Paleontol. J., 49: 13831412.

Mabrouki, Y.; Taybi, A.F.; Chavanon, G.; Berrahou, A. and Millán, A. (2018). Distribution of aquatic beetles from the east of Morocco (Coleoptera, Polyphaga). Arxius de Miscel-lània Zoològica, 16: 185-211, Doi: https://doi.org/10.32800/amz.2018.1 $\underline{6.0185}$

Salah, M.; Regil, J. A. and Valladares, L.F. (2014). An annotated checklist of the aquatic Polyphaga (Coleoptera) of Egypt I. Family Hydraenidae. Zootaxa, 3873(3): 275-284.

Ślipiński. A.; Leschen, R. and Lawrence, J. (2011). Order Coleoptera Linnaeus, 1758. In: Zhang, Z.-Q. (Ed), Animal biodiversity: An outline of higherlevel classification and survey of taxonomic richness. Zootaxa, 3148: 203-208.

Touaylia, S.; Garrido, J.; Bejaoui, M. and Boumaiza, M. (2011). Altitudinal distribution of aquatic beetles (Coleoptera) in Northern Tunisia: Relationship between Species Richness and Altitude. ,65(1): 5362, Doi: https://doi:10.1649/0010$\underline{065 x-65.1 .537}$ 


\section{Ahmed R. Ismaieel et al.}

\section{قائمه مرجعيه تفصيليه لفصيله هيدرينيدي ( غمديه الاجنحه: ستافيلونويديا) في شمال افريقيا

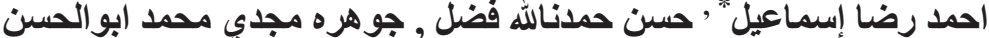

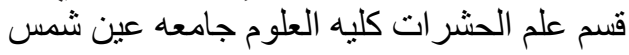 \\ *ahmedreda@sci.asu.edu.eg}

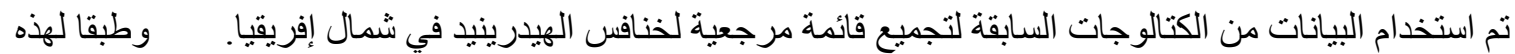

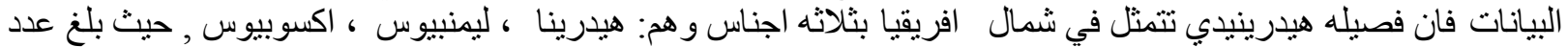

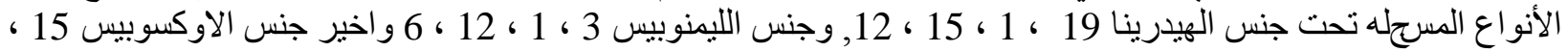

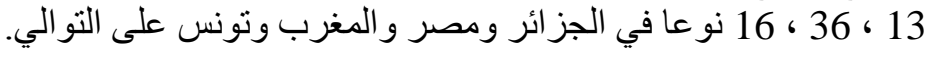

